\title{
A Non-Linear Association of Triglyceride Glycemic Index With Cardiovascular and All-Cause Mortality Among Patients With Hypertension
}

\section{OPEN ACCESS}

Edited by:

Modar Kassan,

University of Tennessee Health

Science Center (UTHSC),

United States

Reviewed by:

Yang Peng,

The University of

Queensland, Australia

Masahide Hamaguchi,

Kyoto Prefectural University of

Medicine, Japan

*Correspondence:

Ying-qing Feng

1250780250@qq.com

tThese authors have contributed equally to this work

Specialty section:

This article was submitted to

Hypertension,

a section of the journal

Frontiers in Cardiovascular Medicine

Received: 16 September 2021 Accepted: 27 December 2021

Published: 27 January 2022

Citation:

Zhou D, Liu X-c, Kenneth L, Huang $Y$-q and Feng Y-q (2022) $A$ Non-Linear Association of Triglyceride

Glycemic Index With Cardiovascular and All-Cause Mortality Among

Patients With Hypertension.

Front. Cardiovasc. Med. 8:778038.

doi: 10.3389/fcvm.2021.778038

\begin{abstract}
Dan Zhou ${ }^{1+}$, Xiao-cong Liu ${ }^{1 \dagger}$, Lo Kenneth ${ }^{1,2,3}$, Yu-qing Huang ${ }^{1}$ and Ying-qing Feng ${ }^{\text {** }}$
1 Department of Cardiology, Guangdong Cardiovascular Institute, Guangdong Provincial People's Hospital, Guangdong Academy of Medical Sciences, Guangzhou, China, ${ }^{2}$ Department of Epidemiology, Centre for Global Cardio-Metabolic Health, Brown University, Providence, Rl, United States, ${ }^{3}$ Department of Applied Biology and Chemical Technology, The Hong Kong Polytechnic University, Hong Kong, Hong Kong SAR, China
\end{abstract}

Background: To investigate the association between insulin resistance (IR), quantified by triglyceride glycemic index (TyG index), cardiovascular mortality (CVM), and all-cause mortality (ACM) in hypertension patients.

Methods: We included 8,554 patients with hypertension aged $\geq 18$ years old from the 1999-2014 National Health and Nutrition Examination Surveys (NHANES). The status of CVM and ACM of participants were followed through December 31, 2015. Cox proportional hazards models and Kaplan-Meier survival curves were used to evaluate the relationship between TyG index, CVM, and ACM.

Results: During a median of 82 months follow-up, 1,882 mortality cases had occurred, 434 of which were due to cardiovascular disease. The patients with hypertension with TyG $\geq 10$ were older, had a higher chance of being smokers, were obese, had higher blood pressure, and had risk or had cardiovascular disease. In Cox proportional hazards models, compared with the patients with $\mathrm{TyG}<8$, those with TyG $\geq 10$ had 56\% increased risk for ACM. On the other hand, no significant difference for CVM between the four groups were observed. In the restricted cubic spline regression models, the relationship between TyG index and ACM was non-linear. Subgroup analysis showed non-linear relationship between TyG index and ACM in elderly patients aged $\geq 60$ years. The cut-off value of TyG for ACM was 9.45, and those with higher or lower than 9.45 had more risk of ACM. When TyG index was more than 9.52, the risk for CVM would increase among the whole group. Kaplan-Meier survival curves showed patients with TyG $\geq 10$ had higher risk of ACM and CVM (Log rank $P<0.05)$.

Conclusions: We demonstrated that the association between ACM and TyG index in elderly patients with hypertension aged $\geq 60$ years was non-linear. However, TyG index was only more than 9.52, hence, the risk for CVM would increase among the whole hypertension group.

Keywords: insulin resistance, triglyceride-glucose index, hypertension, all-cause mortality, cardiovascular mortality 


\section{BACKGROUND}

Hypertension is a primary risk factor for cardiovascular disease and all-cause mortality (ACM) (1, 2). Careful risk factor assessment plays an important role in hypertension patients. The metabolic effects of insulin resistance (IR), including hyperglycemia and dyslipidemia, appear to interact synergistically with increased blood pressure to cause vascular and kidney injury (3). IR is proposed to be important contributors to hypertension-caused organ damage, arterial stiffness (4), and left ventricular diastolic dysfunction (5) due to hypertension. Hence, higher IR is a risk factor for hypertension patients.

At present, Homeostatic model assessment IR (HOMA-IR), a method for assessing $\beta$-cell function and IR, is frequently applied, but its high cost and inconvenience make it difficult to use in large

Abbreviations: IR, insulin resistance; CVM, cardiovascular mortality; ACM, allcause mortality; TyG index, triglyceride glycemic index; HOMA-IR, homeostatic model assessment IR; SBP, systolic blood pressure; DBP, diastolic blood pressure; TC, total cholesterol; TG, triglyceride; LDL-C, low-density lipoprotein cholesterol; HDL-C, high-density lipoprotein cholesterol; FBG, fasting blood glucose; eGFR, estimated glomerular filtration rate; BMI, body mass index; HbA1C, hemoglobin Alc cohort study. Therefore, there has been no large cohort study examinate the prognosis value of IR in hypertension group.

Triglyceride-Glucose index (TyG index), first published by South American authors, showed a good correlation with the insulin clamp technique and HOMA-IR index. The TyG index, described to be a simple, convenient, and low-cost method, is of research interest in many countries in Asia and can be used to screen for IR in the Asian hypertensive community (6). Despite this, there is no evidence between TyG index and prognosis value in North America hypertensive community. Therefore, we conducted a retrospective cohort study to certify the relationship of cardiovascular mortality (CVM) and ACM in hypertension patients.

\section{METHODS}

\section{Study Population}

The National Health and Nutrition Examination Surveys (NHANES), sponsored by the Centers for Disease Control and Prevention, is designed to assess the health status of US citizens. Data for analyses were taken from the NHANES 1999-2014 with a total of 82,091 participants. In our analysis, we included people aged $\geq 18$ years old. Subjects without blood lipid data,

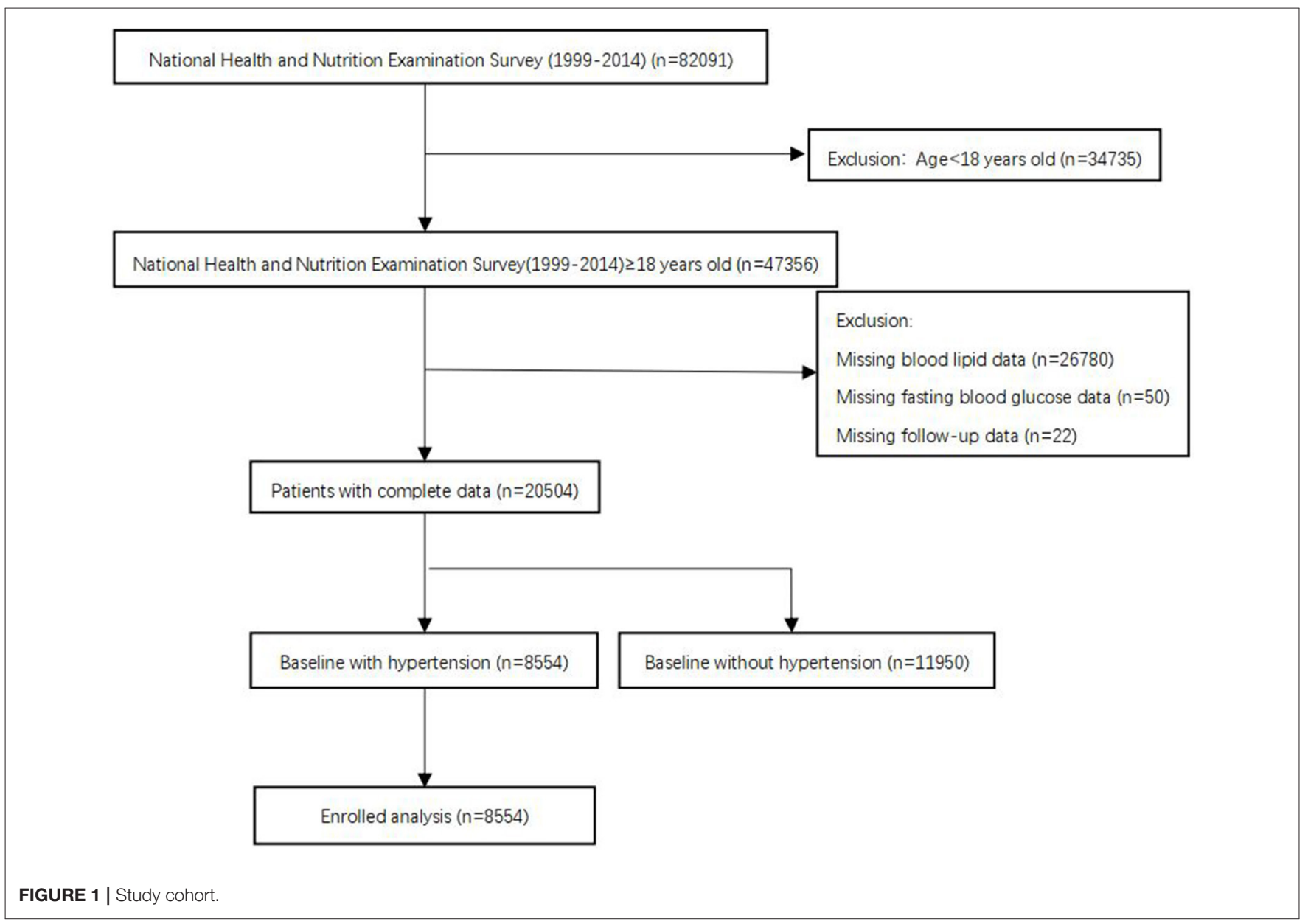


TABLE 1 | Demographic and clinical characteristics according to triglyceride-glucose index level.

\begin{tabular}{|c|c|c|c|c|c|c|}
\hline & Total & TyG $<8$ & $8 \leq \mathrm{T} y \mathrm{G}<9$ & $9 \leq$ TyG $<10$ & TyG $\geq 10$ & $p$ \\
\hline Number & 8,554 & 690 & 4,655 & 2,743 & 466 & \\
\hline Age, years & $60.12 \pm 16.07$ & $54.13 \pm 18.33$ & $60.70 \pm 16.32$ & $60.92 \pm 15.01$ & $58.50 \pm 13.96$ & $<0.001$ \\
\hline Male & $4,216(49.29)$ & 327 (47.39) & $2,203(47.33)$ & $1,412(51.48)$ & $274(58.80)$ & \\
\hline Female & $4,338(50.71)$ & 363 (52.61) & $2,452(52.67)$ & $1,331(48.52)$ & $192(41.20)$ & \\
\hline White & $4,309(50.37)$ & 258 (37.39) & $2,320(49.84)$ & $1,505(54.87)$ & $226(48.50)$ & \\
\hline Marital status, $\mathbf{n}(\%)$ & & & & & & $<0.001$ \\
\hline Married & 4,632 (54.91) & $329(48.53)$ & 2,493 (54.31) & 1,552 (57.29) & $258(56.33)$ & \\
\hline Other & $3,803(45.09)$ & $349(51.47)$ & 2,097 (45.69) & $1,157(42.71)$ & $200(43.67)$ & \\
\hline Education level, $\mathbf{n}(\%)$ & & & & & & $<0.001$ \\
\hline Yes & $4,280(50.60)$ & $301(45.20)$ & 2,239 (48.62) & $1,473(54.07)$ & $267(57.67)$ & \\
\hline Body mass index, $\mathrm{kg} / \mathrm{m}^{2}$ & $30.31 \pm 7.09$ & $28.11 \pm 7.30$ & $29.76 \pm 7.26$ & $31.49 \pm 6.51$ & $32.15 \pm 6.80$ & $<0.001$ \\
\hline Systolic blood pressure, $\mathrm{mmHg}$ & $135.62 \pm 21.35$ & $133.21 \pm 20.58$ & $135.11 \pm 21.62$ & $136.53 \pm 20.96$ & $138.89 \pm 21.55$ & $<0.001$ \\
\hline Diastolic blood pressure, $\mathrm{mmHg}$ & $71.00 \pm 16.07$ & $71.07 \pm 16.05$ & $70.44 \pm 16.21$ & $71.79 \pm 15.69$ & $71.93 \pm 16.69$ & 0.004 \\
\hline eGFR, $\mathrm{mg} / \mathrm{min} / 1.73 \mathrm{~m}^{2}$ & $79.31 \pm 26.93$ & $86.21 \pm 27.81$ & $79.29 \pm 26.98$ & $77.24 \pm 25.85$ & $81.55 \pm 29.48$ & $<0.001$ \\
\hline Total cholesterol, mg/dL & $197.68 \pm 43.43$ & $179.68 \pm 39.45$ & $193.14 \pm 40.25$ & $204.94 \pm 43.47$ & $226.94 \pm 56.40$ & $<0.001$ \\
\hline HDL cholesterol, mg/dL & $52.87 \pm 16.31$ & $66.46 \pm 19.88$ & $56.03 \pm 15.75$ & $46.36 \pm 12.26$ & $39.46 \pm 11.65$ & $<0.001$ \\
\hline LDL cholesterol, mg/dL & $115.31 \pm 36.57$ & $102.64 \pm 32.30$ & $116.05 \pm 35.35$ & $117.83 \pm 38.74$ & $109.09 \pm 38.95$ & $<0.001$ \\
\hline Triglycerides, mg/dL & $150.58 \pm 117.66$ & $52.23 \pm 11.80$ & $104.85 \pm 28.96$ & $203.81 \pm 65.30$ & $439.76 \pm 291.68$ & $<0.001$ \\
\hline Fasting blood glucose, mg/dL & $115.87 \pm 42.86$ & $92.76 \pm 12.14$ & $104.34 \pm 18.47$ & $125.30 \pm 40.63$ & $209.87 \pm 94.41$ & $<0.001$ \\
\hline TyG index & $8.86 \pm 0.68$ & $7.76 \pm 0.21$ & $8.56 \pm 0.27$ & $9.37 \pm 0.26$ & $10.51 \pm 0.47$ & $<0.001$ \\
\hline \multicolumn{7}{|l|}{ Treatment, n (\%) } \\
\hline Antihypertensive drugs & & & & & & $<0.001$ \\
\hline No & $3,254(38.04)$ & $333(48.26)$ & 1,854 (39.83) & $919(33.50)$ & $148(31.76)$ & \\
\hline Yes & $5,300(61.96)$ & 357 (51.74) & $2,801(60.17)$ & $1,824(66.50)$ & $318(68.24)$ & \\
\hline Hypoglycemic agents & & & & & & $<0.001$ \\
\hline No & $7,115(83.18)$ & $647(93.77)$ & $4,149(89.13)$ & $2,089(76.16)$ & $230(49.36)$ & \\
\hline Yes & 1,439 (16.82) & $43(6.23)$ & $506(10.87)$ & $654(23.84)$ & 236 (50.64) & \\
\hline Lipid-lowering drugs & & & & & & $<0.001$ \\
\hline No & 6,309 (73.75) & 569 (82.46) & $3,498(75.15)$ & 1,936 (70.58) & $306(65.67)$ & \\
\hline Yes & $2,245(26.25)$ & $121(17.54)$ & $1,157(24.85)$ & $807(29.42)$ & $160(34.33)$ & \\
\hline Antiplatelet drugs & & & & & & 0.024 \\
\hline No & 8,185 (95.69) & 666 (96.52) & 4,476 (96.15) & $2,600(94.79)$ & $443(95.06)$ & \\
\hline Yes & $369(4.31)$ & $24(3.48)$ & $179(3.85)$ & $143(5.21)$ & $23(4.94)$ & \\
\hline \multicolumn{7}{|l|}{ Outcomes, n (\%) } \\
\hline Cardiovascular disease mortality & & & & & & $<0.001$ \\
\hline No & $8,120(94.93)$ & 659 (95.51) & 4,445 (95.49) & 2,595 (94.60) & $421(90.34)$ & \\
\hline Yes & $434(5.07)$ & $31(4.49)$ & $210(4.51)$ & $148(5.40)$ & $45(9.66)$ & \\
\hline All-cause mortality & & & & & & $<0.001$ \\
\hline No & 6,672 (78.00) & $582(84.35)$ & $3,650(78.41)$ & $2,125(77.47)$ & $315(67.60)$ & \\
\hline Yes & $1,882(22.00)$ & $108(15.65)$ & $1,005(21.59)$ & $618(22.53)$ & $151(32.40)$ & \\
\hline
\end{tabular}

$Q$, quintiles; $n$, number; $H D L$, high density lipoprotein; $L D L$, low density lipoprotein; TyG, Triglyceride-glucose. Values are mean \pm standardized differences or $n(\%)$. 
TABLE 2 | Multivariate Cox regression analysis of triglyceride-glucose index with cause-specific mortality.

\begin{tabular}{|c|c|c|c|c|}
\hline & Event rate/1,000 person-years & Model I & Model II & Model III \\
\hline \multicolumn{5}{|c|}{ All-cause mortality } \\
\hline TyG $<8$ & 24.07 & 1.0 & 1.0 & 1.0 \\
\hline $8 \leq \operatorname{TyG}<9$ & 29.16 & $1.19(0.98,1.45), 0.0841$ & 0.84 (0.69, 1.02), 0.0794 & $0.90(0.72,1.13), 0.3626$ \\
\hline P for trend & & $<0.001$ & 0.002 & 0.099 \\
\hline \multicolumn{5}{|c|}{ Cardiovascular mortality } \\
\hline \multicolumn{5}{|c|}{ TyG index group } \\
\hline TyG $<8$ & 6.91 & 1.0 & 1.0 & 1.0 \\
\hline $8 \leq \operatorname{TyG}<9$ & 6.09 & $0.87(0.60,1.27), 0.4776$ & 0.62 (0.42, 0.90), 0.0125 & $0.55(0.36,0.82), 0.0039$ \\
\hline
\end{tabular}

TyG, triglyceride-glucose; HR, hazard ratio; Cl, confidence interval; Q, quintiles.

Model I adjust for none.

Model II adjust for age, gender, and race.

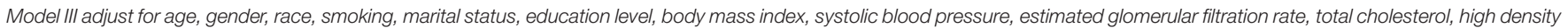

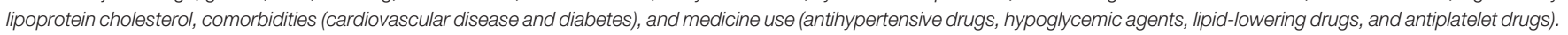

fasting blood glucose data, follow-up data, or baseline without hypertension were excluded. After applying the criteria, we enrolled 8,554 participants for final analysis (Figure 1). The survival status of participants was followed up to December 31, 2015. The NHANES study protocol was approved by the Institutional Review Board of the Centers for Disease Control and Prevention. Informed consent was signed by all participants.

\section{Data Collection}

Questionnaires were collected at baseline to acquire demographic information (age, gender, race, marital status, and education level), smoking status, personal medical history (cardiovascular diseases and diabetes), and medication history (antihypertensive drugs, hypoglycemic agents, lipid-lowering drug, and antiplatelet drugs).

Physical assessments were performed to examine height, weight, systolic blood pressure (SBP), and diastolic blood pressure (DBP). Blood samples were collected on an empty stomach in the morning and after $8 \mathrm{~h}$. Total cholesterol (TC), triglyceride (TG), low-density lipoprotein cholesterol (LDL-C), high-density lipoprotein cholesterol (HDL-C), fasting blood glucose (FBG), and estimated glomerular filtration rate (eGFR) were also collected. Body mass index (BMI) was calculated using weight $(\mathrm{kg})$ divided by the square of height $\left(\mathrm{m}^{2}\right)$. Hypertension was defined as having an SBP $\geq 140$ or/and DBP $\geq 90 \mathrm{mmHg}$, is confirmed to be taking antihypertensive medications, or has a self-reported history of hypertension (7). Diabetes was defined as having an FBG $\geq 126 \mathrm{mg} / \mathrm{dl}$, having a self-reported hemoglobin $\mathrm{A} 1 \mathrm{c}(\mathrm{HbA1C}) \geq 6.5 \%$, or using hypoglycemic drug (8). TyG index was calculated by $\ln$ [fasting triglycerides $(\mathrm{mg} / \mathrm{dl}) *$ fasting glucose ( $\mathrm{mg} / \mathrm{dl}) / 2]$ (9). eGFR was computed using Modification of Diet in Renal Disease (MDRD) formula (10).

\section{Clinical Outcome}

All-cause mortality refer to death from any cause, cardiovascular disease, or cerebrovascular disease until December 31, 2015 were the primary outcomes. Mortality data were extracted from the 1999-2014 NHANES public-use linked mortality files. We examined the time from enrollment (date of interview) to mortality for censoring. The International Classification of Diseases, Tenth Revision codes (I00-I09, I11, I13, I20-I51) were used to define cardiovascular deaths. Any participant who was not matched with any death records was considered to be alive throughout the follow-up period.

\section{Statistical Analysis}

Continuous variables were expressed as mean \pm standard deviation for normally distributed variables or median (interquartile range) if the data were not normally distributed. Categorical variables were presented as number (n) and percentage (\%). The one-way ANOVA, Kruskal-Wallis $H$-test or chi-square tests were used to assess differences according to baseline TyG index $($ TyG $<8,8 \leq$ TyG $<9,9 \leq$ TyG $<10$, TyG $\geq 10$ ) in groups. Multivariable Cox regression analysis were used to estimate adjusted hazard ratio (HR) and 95\% CI for mortality according to baseline TyG index in groups. Model I adjust for none. Model II adjust for age, gender, and race. Age, gender, race, smoking, marital status, education level, BMI, SBP, eGFR, TC, HDL-C, comorbidities (cardiovascular disease and diabetes), and medicine use (antihypertensive drugs, hypoglycemic agents, lipid-lowering drugs, and antiplatelet drugs) were included in Model III. We initially performed survival analysis using standardized Kaplan-Meier curves and Log rank test. The association between TyG index, ACM, and CVM was then examined by multivariate adjusted Cox restricted 

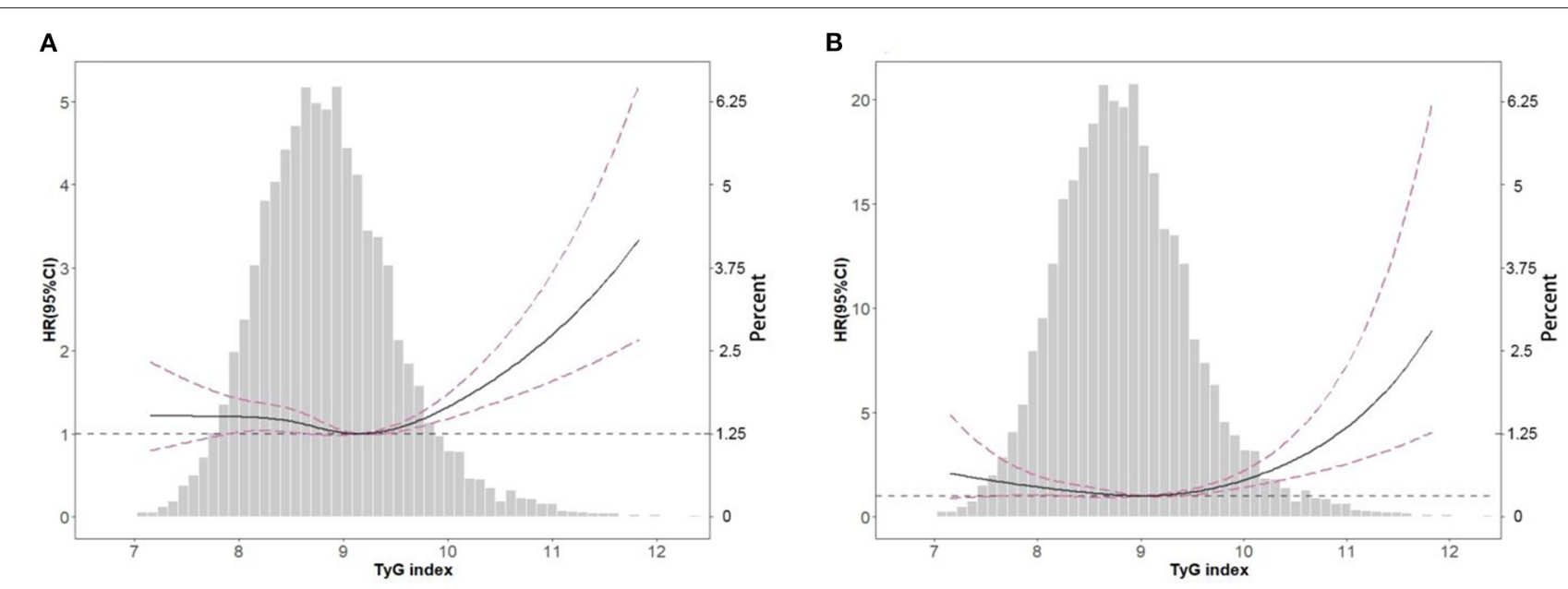

FIGURE 2 | Spline analyses of all-cause (A) and cardiovascular (B) mortality by TyG index in the overall cohort and the probability distribution histogram is represented in the background (spline analyses were adjusted for age, gender, race, smoking, marital status, education level, body mass index, systolic blood pressure, estimated glomerular filtration rate, total cholesterol, high density lipoprotein cholesterol, cardiovascular disease, diabetes, antihypertensive drugs, hypoglycemic agents,

lipid-lowering drugs, and antiplatelet drugs).

TABLE 3 | The results of two-piecewise linear regression model between triglyceride-glucose index and cause-specific mortality.

\begin{tabular}{|c|c|c|}
\hline & $\begin{array}{l}\text { All-cause mortality } \\
\text { HR ( } 95 \% \mathrm{Cl}), p \text { value }\end{array}$ & $\begin{array}{c}\text { Cardiovascular disease } \\
\text { mortality } \\
\text { HR }(95 \% \mathrm{Cl}), p \text { value }\end{array}$ \\
\hline Cutoff value & 9.45 & 9.52 \\
\hline$<$ Cut-off value & 0.87 (0.76, 0.99), 0.0329 & 0.81 (0.62, 1.06), 0.1277 \\
\hline$\geq$ Cut-off value & $1.73(1.44,2.09),<0.0001$ & $2.85(2.05,3.98),<0.0001$ \\
\hline $\begin{array}{l}p \text { for log likelihood ratio } \\
\text { test }\end{array}$ & $<0.001$ & $<0.001$ \\
\hline
\end{tabular}

TyG, triglyceride-glucose; HR, hazard ratio; Cl, confidence interval.

The two-piecewise linear regression model were adjusted for age, gender, race, smoking, marital status, education level, body mass index, systolic blood pressure, estimated glomerular filtration rate, total cholesterol, high density lipoprotein cholesterol, comorbidities (cardiovascular disease and diabetes), and medicine use (antihypertensive drugs, hypoglycemic agents, lipid-lowering drugs, and antiplatelet drugs).

cubic spline regression models and used a generalized additive model to explore the non-linear relationship between TyG index and mortality. If non-linear relationships were identified, we used two-piecewise linear regression models to elucidate how the associations differed by the threshold point. The threshold value was estimated by trying all possible value and choosing the threshold point with highest likelihood. Finally, we conducted subgroup analyses, including age ( $<65$ or $\geq 65$ years), gender (male or female), race (white or non-white), and BMI ( $<25$ or $\geq 25 \mathrm{~kg} / \mathrm{m}^{2}$ ). $P<0.05$ was considered statistically significant. R version 3.3.2 (R Foundation for Statistical Computing, Vienna, Austria) was used for all statistical analyses.

\section{RESULTS}

\section{Baseline Characteristics}

The baseline characteristics of the analytical cohort according to TyG index in groups were showed in Table 1. In total,
8,554 patients $(49.29 \%$ men) were included in this analysis with mean age of $60.12 \pm 16.07$ years. Of these, $4,309(50.37 \%)$ participants were white, 4,632 (54.91\%) married, 5,716 (67.70\%) received high school or above education, and 4,178 (49.40\%) never smoked. The patients with hypertension in group of TyG $\geq 10$ were older, were more likely to be smokers, were obese, had higher blood pressure, and had higher risk or had cardiovascular disease. In addition, the proportion of participants with diabetes and cardiovascular disease was 30.33 and $19.36 \%$, respectively. During a median follow up of 82 months, 1,882 cases of death have occurred, 434 of which were due to cardiovascular disease. All baseline variables differed significantly among the TyG index in groups (all $p<0.05$ ).

\section{Hazard Ratios (HRs) of TyG Index for ACM and CVM Risk}

Table 2 reveals the estimated HRs and CIs of TyG index in relation to ACM and CVM. In the Model III, compared with the lowest group of TyG index (TyG $<8$ ), the HRs for ACM from other groups $(8 \leq \mathrm{TyG}<9,9 \leq \mathrm{TyG}<10$, TyG $\geq 10)$ in the fully adjusted model were $0.90(0.72$ to $-1.13, p=0.36), 0.85(0.66-$ $1.10, p=0.21)$, and $1.56(1.14-2.15, p<0.05)$, respectively $(P$ for trend $=0.09$ ).

Population with TyG $\geq 10$ showed a $56 \%$ increased risk of ACM compared with those TyG $<8$. After an adjustment for confounders, the HRs and CIs for CVM from the three groups $(8 \leq$ TyG $<9,9 \leq$ TyG $<10$, TyG $\geq 10)$ were $0.55(0.36,0.82)$, $0.53(0.33,0.86)$, and $1.23(0.67,2.25)$, respectively $(P$ for trend $=$ 0.43). After excluding type 2 diabetes mellitus or cardiovascular disease in the baseline, in Supplementary Table 1, we found that when comparing with the lowest group of TyG index $(\mathrm{TyG}<8)$, the HRs for ACM from TyG $\geq 10$ in the fully adjusted model were $2.05(1.04,4.04)(p=0.03)$. However, it was not associated with any change in the risk of CVM.

In the restricted cubic spline regression models with full adjustment for confounders, the relationships between TyG 


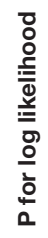

\section{$\overline{8}$ \\ 5 \\ ธo̊ \\ $\overline{8}$
$\dot{0}$
$\dot{0}$
V}

웅

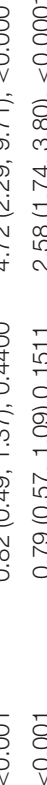

$\overline{8}$

$\begin{array}{ll}\overline{8} & \overline{8} \\ 0 & 8 \\ 0 & 0\end{array}$

\begin{tabular}{ll}
$\overline{8}$ & $\overline{8}$ \\
$\circ$ & 8 \\
\hline & 0
\end{tabular}

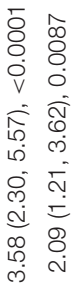

它

E

00

के ले

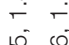

कृ की

을

กิ กิ

$\begin{array}{llll}0 & 0 \\ 0 & 0 & 0 \\ 0 & 0 & 0\end{array}$

0

웜

₹

อ 9

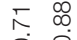

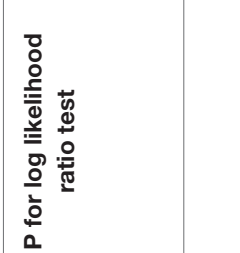

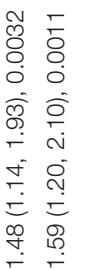

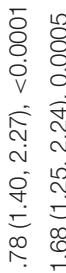

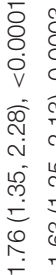

$\overline{8} \overline{8}$

०ं

के बू

$\dot{+} \dot{-}$

क)

$\stackrel{2}{\infty}=$ index, ACM (Figure 2A), and CVM (Figure 2B) were both nonlinear in participants with hypertension. The results of two piecewise linear regression model are demonstrated in Table 3. The relationship between TyG index and ACM was non-linear. Subgroup analysis (Table 4) showed non-linear relationship between TyG index and ACM in elderly patients with aged $\geq 60$ years. The cut-off value of TyG for ACM was 9.45. Values higher or lower than 9.45 had more risk of ACM. When TyG index was more than 9.52, the risk for CVM would increase among the whole group.

As showed in Kaplan-Meier survival curves (Figure 3), hypertension patients with $\mathrm{TyG} \geq 10$ had significantly higher ACM (Figure 3A) and CVM (Figure 3B) in the following life (log rank $p<0.05)$.

\section{Subgroup Analyses}

Table 4 has explored the relationship between TyG index, ACM, and CVM as stratified by gender, age, BMI, and race. Only for age $\geq 60$ years old patients, the association was significant different between TyG index $<9.45$ or $\geq 9.45$. When index $\geq 9.45$, risk increased by $48 \%$ at every $1 \mathrm{SD}$ increase of TyG index. When index $<9.45$, risk increased by $19 \%$ at every 1 SD decrease of TyG index. Other subgroups only showed a higher TyG index, more than the cut-off value, would present higher risk for ACM and CVM. When TyG index was less than the cut-off value, it showed no difference.

\section{DISCUSSION}

In this retrospective study, we, for the first time, revealed association between IR (TyG index), ACM, and CVM among patients with hypertension in a large cohort study. We demonstrated that the association was non-linear between ACM and TyG index in elderly patient with aged $\geq 60$ years old. But for CVM, only when TyG index was more than 9.52 will the risk for CVM increase among the whole hypertension group.

We also demonstrated the association was non-linear between ACM and TyG index in elderly with aged $\geq 60$ years old. When TyG index $\geq 9.45$, the risk of ACM increased by $48 \%$ at every $1 \mathrm{SD}$ increase of TyG index. In contrast, for TyG index $<9.45$, the risk of ACM increased by $19 \%$ as the TyG index decreased by $1 \mathrm{SD}$. TyG index, a composite indicator based on TG level and FBG value, was shown to be used as a surrogate marker of IR $(9,11,12)$. Previous studies probed the relationship between IR and ACM in different population. An observational prospective cohort study that enrolled 15,773 patients with type 2 diabetes showed that highest IR predicts ACM in type 2 diabetes (13). High IR, as measured by HOMAIR, identified postmenopausal women at higher risk for cancerspecific and ACM (14). In addition, A meta-analysis included seven articles involving 26,976 non-diabetic adults showed that the highest HOMA-IR increased the risk of ACM by $34 \%$ when compared with the lowest category (15). For elderly people, there were different conclusions. Among community-dwelling older individuals over 65 years, HOMA-IR and low-grade systemic inflammation was associated with a 9-year ACM and CVM risk (16). Aging and high C-reactive protein levels were, usually, 
A

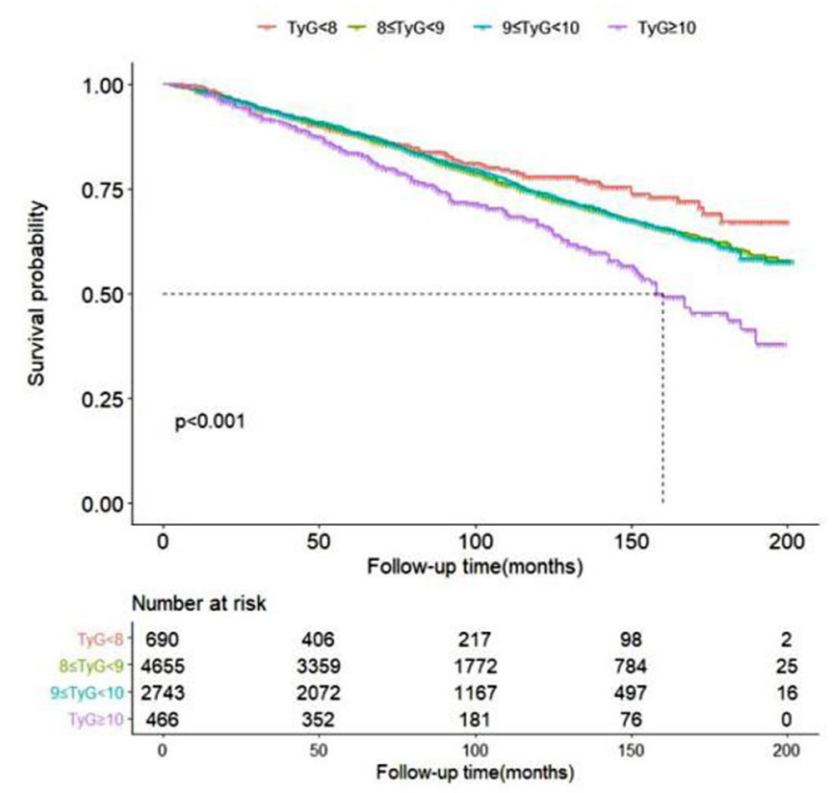

B

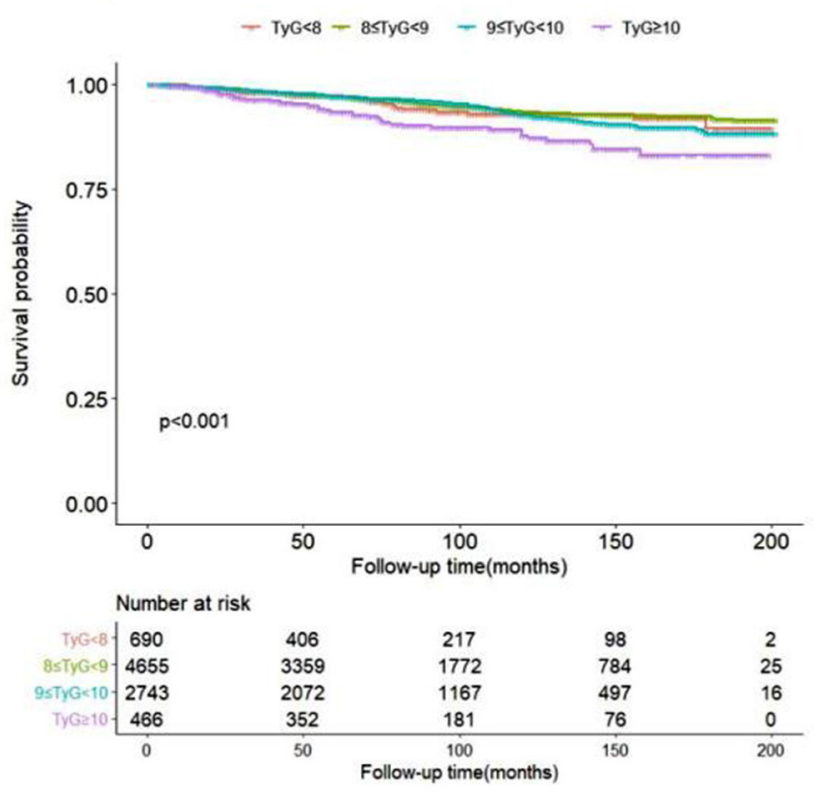

FIGURE 3 | Kaplan-Meier survival curve for all-cause (A) and cardiovascular (B) mortality by TyG index.

risk factors for cardiovascular disease mortality. We did not evaluate systemic inflammation in our study. A communitybased prospective study selected participants with age $\geq 65$ years in Korea (17). Elderly subjects in the fifth and first quintile of HOMA-IR values had increased rates of ACM and CVM as there is a threshold level of HOMA-IR in relation to mortality. The HOMA-IR was lower in Korea study (1st quintile $\leq 0.67$ and 5 th quintile $>1.50$ ). Our work showed similar with the study in Korea; however, we used the TyG index to evaluate IR, not HOMA-IR, in elderly hypertension patients.

A previous study showed that there was a striking U-shaped relationship between FBG levels and in-hospital and 3-year mortality in older patients with acute myocardial infarction (AMI) (18). The study observed that mild to moderately low FBG levels $(\leq 5 \mathrm{mmol} / \mathrm{L}, 90 \mathrm{mg} / \mathrm{dl})$ were associated with a relative increase in mortality risk. Hypoglycemia and rapid changes in blood glucose level were shown to increase levels of counterregulatory hormones, such as epinephrine and norepinephrine, which may induce vasoconstriction and platelet aggregation, resulting in ischemia of cardiovascular or cerebrovascular (19). In the elderly population, the decrease in skeletal muscle mass is generally accompanied by weight loss. A study that recruited elderly individuals ( $\geq 65$ years) in Taiwan showed that elderly subjects with sarcopenic obesity had the highest ACM (19). In contrast, except for the TG level, none of the other lipid profile indices were related to ACM in patients aged over 75 years (20). The mortality risk decreased by $17 \%$ for each $1 \mathrm{mmol} / \mathrm{L}$ increase serum level of TG in ZODIAC study (20). The lower TG had higher risk of ACM for elderly population. One study, which enrolled individuals aged 65 and older residing in northern Manhattan in 1992-1994 and 19992002, showed low cholesterol level was a robust predictor of mortality in the non-demented elderly and may be a surrogate of frailty or subclinical disease (21). Our study was collected from 1999 to 2014 in the same country as the study (21), so the life diet structure and level were similar. The mean TG of the patients with mortality was $155 \mathrm{mg} / \mathrm{dl}$ (21). Therefore, the finding of our study, specifically of the $1 \mathrm{SD}$ decrease of TyG index when TyG index $<9.45$ in elderly with aged $\geq 60$ years and $19 \%$ increased risk of ACM was persuasive. Other factors, such as body composition and nutritional state, might be predictors of mortality in the elderly population (17). Older age, anemia, lower baseline hemoglobin level (22), and lower BMI had a greater effect on mortality.

Only when TyG index $\geq 9.52$, TyG-index showed significant with CVM. A previous study had reported that IR was associated with cardiovascular disease (23). The relationship between IR and endothelial dysfunction may the underlying biological mechanism of cardiovascular. This mostly includes inflammation (24) and functional impairment in the endothelium of blood vessels (25) which leads to atherosclerosis and cardiovascular disease. This simple, convenient, and low-cost TyG index was of research interest in many Countries and could be used to screen for IR in the hypertensive community. Furthermore, Park et al. (26) reported that TyG-index is an independent predictor of coronary artery calcium progression. We first investigate relationship between TyG index and CVM and ACM in hypertension. The Kaplan-Meier survival curve by TyG index groups also confirmed that the higher TyG index was associated with increased higher incident of ACM and CVM. 
Our study still had several limitations that need to be mentioned. First, we did not have multiple-time monitoring of the TyG index along the follow-up, which may provide more information. Secondly, we did not compare the TyG index with HOMA-IR and the hyperinsulinemic-euglycemic clamp test. Thirdly, nutritional habits or energy intake were not recorded. Although we did not adjust for these potential confounding variables, we used other variables, such as BMI or cholesterol levels, which are indirectly related to nutritional habits or energy intake. Finally, this was a population-based study conducted among hypertension participants in the United States. Therefore, our findings may not be generalizable to other populations.

\section{CONCLUSION}

We first time revealed association between IR (TyG index), ACM, and CVM among patients with hypertension. We demonstrated that the association was non-linear between ACM and TyG index in elderly with aged $\geq 60$ years. But for CVM, higher TyG index had high risk among patients with hypertension only when TyG index $\geq 9.52$.

\section{DATA AVAILABILITY STATEMENT}

The datasets presented in this study can be found in online repositories. The names of the repository/repositories and accession number(s) can be found in the article/Supplementary Material.

\section{ETHICS STATEMENT}

The studies involving human participants were reviewed and approved by the Institutional Review Board of the Centers for Disease Control and Prevention. The patients/participants provided their written informed consent to participate in this study.

\section{REFERENCES}

1. Kjeldsen SE, Narkiewicz K, Burnier M, Oparil S. The global burden of disease study 2015 and blood pressure. Blood Press. (2017) 26:1. doi: 10.1080/08037051.2016.1267557

2. Bromfield S, Muntner P. High blood pressure: the leading global burden of disease risk factor and the need for worldwide prevention programs. Curr Hypertens Rep. (2013) 15:134-6. doi: 10.1007/s11906-013-0340-9

3. da Silva AA, do Carmo JM, Li X, Wang Z, Mouton AJ, Hall JE. Role of hyperinsulinemia and insulin resistance in hypertension: metabolic syndrome revisited. Can J Cardiol. (2020) 36:671-82. doi: 10.1016/j.cjca.2020. 02.066

4. Bello-Chavolla OY, Antonio-Villa NE, Vargas-Vázquez A, Martagón AJ, Mehta R, Arellano-Campos O, et al. Prediction of incident hypertension and arterial stiffness using the non-insulin-based metabolic score for insulin resistance (METS-IR) index. J Clin Hypertens. (2019) 21:106370. doi: $10.1111 /$ jch.13614

5. Bamaiyi AJ, Woodiwiss AJ. Insulin resistance influences the impact of hypertension on left ventricular diastolic dysfunction in a community sample. Clin Cardiol. (2019) 42:305-11. doi: 10.1002/clc.23145

\section{AUTHOR CONTRIBUTIONS}

$\mathrm{DZ}$ and $\mathrm{Y}-\mathrm{qF}$ contributed to the conception or design of the study and drafted the manuscript. X-cL contributed to the acquisition of data, interpretation of data, and analysis of data. $\mathrm{Y}-\mathrm{qH}$ and $\mathrm{LK}$ contributed to the interpretation of data and critical revision of the article for important intellectual content. All authors gave final approval of the article.

\section{FUNDING}

This research was supported by Science and Technology Plan Program of Guangzhou (201803040012), the Key Area R\&D Program of Guangdong Province (No. 2019B020227005), Guangdong Provincial People's Hospital Clinical Research Fund (Y012018085), the Fundamental and Applied Basic Research Foundation Project of Guangdong Province (2020A1515010738), High-level Hospital Construction Project of Guangdong Provincial People's Hospital (DFJH2020022), and Guangdong Provincial Clinical Research Center for Cardiovascular Disease (2020B111 1170011).

\section{ACKNOWLEDGMENTS}

We gratefully acknowledge the contributions of all staffs who work on the National Health and Nutrition Examination Surveys.

\section{SUPPLEMENTARY MATERIAL}

The Supplementary Material for this article can be found online at: https://www.frontiersin.org/articles/10.3389/fcvm. 2021.778038/full\#supplementary-material

6. Minh HV, Tien HA, Sinh CT, Thang DC, Chen CH. Assessment of preferred methods to measure insulin resistance in Asian patients with hypertension. $J$ Clin Hypertension. (2021) 23:529-37. doi: 10.1111/jch.14155

7. Chobanian AV, Bakris GL, Black HR, Cushman WC, Green LA, Izzo JL Jr., et al. The seventh report of the joint national committee on prevention, detection, evaluation, and treatment of high blood pressure: the JNC 7 report. JAMA. (2003) 289:2560-72. doi: 10.1001/jama.289.19.2560

8. American Diabetes Association. Standards of medical care for patients with diabetes mellitus. Diab Care. (2003) 26 Suppl 1:S33-50. doi: 10.2337/diacare.26.2007.S33

9. Simental-Mendía LE, Rodríguez-Morán M, Guerrero-Romero F. The product of fasting glucose and triglycerides as surrogate for identifying insulin resistance in apparently healthy subjects. Metab Syndr Relat Disord. (2008) 6:299-304. doi: 10.1089/met.2008.0034

10. Levey AS, Coresh J, Bolton K, Culleton B, Harvey KS, Ikizler TA, et al. K/DOQI clinical practice guidelines for chronic kidney disease: evaluation, classification, and stratification. Am J Kidney Dis. (2002) 39(2 Suppl 1):S1-266.

11. Khan SH, Sobia F, Niazi NK, Manzoor SM, Fazal N, Ahmad F. Metabolic clustering of risk factors: evaluation of triglycerideglucose index (TyG index) for evaluation of insulin resistance. 
Diabetol Metab Syndr. (2018) 10:74. doi: 10.1186/s13098-01 8-0376-8

12. Guerrero-Romero F, Simental-Mendía LE, González-Ortiz M, MartínezAbundis E, Ramos-Zavala MG, Hernández-González SO, et al. The product of triglycerides and glucose, a simple measure of insulin sensitivity. Comparison with the euglycemic-hyperinsulinemic clamp. J Clin Endocrinol Metab. (2010) 95:3347-51. doi: 10.1210/jc.2010-0288

13. Penno G, Solini A, Orsi E, Bonora E, Fondelli C, Trevisan R. et al. Insulin resistance, diabetic kidney disease, and all-cause mortality in individuals with type 2 diabetes: a prospective cohort study. BMC Med. (2021) 19:66. doi: 10.1186/s12916-021-01936-3

14. Pan K, Nelson RA, Wactawski-Wende J, Lee DJ, Manson JE, Aragaki $\mathrm{AK}$, et al. Insulin resistance and cancer-specific and all-cause mortality in postmenopausal women: the women's health initiative. J Natl Cancer Inst. (2020) 112:170-8. doi: 10.1093/jnci/djz069

15. Zhang $\mathrm{X}$, Li J, Zheng $\mathrm{S}$, Luo Q, Zhou C, Wang C. Fasting insulin, insulin resistance, and risk of cardiovascular or all-cause mortality in non-diabetic adults: a meta-analysis. Biosci. Rep. (2017) 37:BSR20170947. doi: 10.1042/BSR20170947

16. Zuliani G, Morieri ML, Volpato S, Maggio M, Cherubini A, Francesconi D, et al. Insulin resistance and systemic inflammation, but not metabolic syndrome phenotype, predict 9 years mortality in older adults. Atherosclerosis. (2014) 235:538-45. doi: 10.1016/j.atherosclerosis.20 14.05.959

17. Kim HJ Ryu J, Ahn SY, Kim S, Lim S, Na KY, et al. Association of insulin resistance with lower glomerular filtration rate and all-cause mortality in the Korean elderly population: a community-based prospective cohort study. Tohoku J Exp Med. (2013) 231:271-9. doi: 10.1620/tjem.231.271

18. Yang SW, Zhou YJ, Nie XM, Liu YY, Du J, Hu DY, et al. Effect of abnormal fasting plasma glucose level on all-cause mortality in older patients with acute myocardial infarction: results from the Beijing Elderly Acute Myocardial Infarction Study (BEAMIS). Mayo Clin Proc. (2011) 86:94104. doi: $10.4065 / \mathrm{mcp} .2010 .0473$

19. Galassetti P, Davis SN. Effects of insulin per se on neuroendocrine and metabolic counter-regulatory responses to hypoglycaemia. Clin Sci. (2000) 99:351-62. doi: 10.1042/cs0990351

20. van Hateren KJ, Landman GW, Kleefstra N, Logtenberg SJ, Groenier KH, Kamper AM, et al. The lipid profile and mortality risk in elderly type 2 diabetic patients: a ten-year follow-up study (ZODIAC-13). PLoS ONE. (2009) 4:e8464. doi: 10.1371/journal.pone.0008464
21. Schupf N, Costa R, Luchsinger J, Tang MX, Lee JH, Mayeux R. Relationship between plasma lipids and all-cause mortality in nondemented elderly. J Am Geriatr Soc. (2005) 53:219-26. doi: 10.1111/j.1532-5415.20 05.53106.x

22. Zakai NA, French B, Arnold AM, Newman AB, Fried LF, Robbins J, et al. Hemoglobin decline, function, and mortality in the elderly: the cardiovascular health study. Am J Hematol. (2013) 88:5-9. doi: 10.1002/ajh.23336

23. Salazar MR, Carbajal HA, Espeche WG, Aizpurúa M, Dulbecco CA, Reaven GM. Comparison of two surrogate estimates of insulin resistance to predict cardiovascular disease in apparently healthy individuals. Nutr Metab Cardiovasc Dis. [2017] 27:366-73. doi: 10.1016/j.numecd.2016.12.002

24. Lee DY, Rhee EJ, Chang Y, Sohn CI, Shin HC Ryu S, et al. Impact of systemic inflammation on the relationship between insulin resistance and all-cause and cancer-related mortality. Postgrad Med. (2018) 81:5262. doi: 10.1016/j.metabol.2017.11.014

25. Ormazabal V, Nair S, Elfeky O, Aguayo C, Salomon C. Association between insulin resistance and the development of cardiovascular disease. Cardiovasc Diabetol. (2018) 17:122. doi: 10.1186/s12933-018-0762-4

26. Park K, Ahn CW, Lee SB, Kang S. Elevated TyG index predicts progression of coronary artery calcification. Diab Care. (2019) 42:156973. doi: $10.2337 / \mathrm{dc} 18-1920$

Conflict of Interest: The authors declare that the research was conducted in the absence of any commercial or financial relationships that could be construed as a potential conflict of interest.

Publisher's Note: All claims expressed in this article are solely those of the authors and do not necessarily represent those of their affiliated organizations, or those of the publisher, the editors and the reviewers. Any product that may be evaluated in this article, or claim that may be made by its manufacturer, is not guaranteed or endorsed by the publisher.

Copyright (๑) 2022 Zhou, Liu, Kenneth, Huang and Feng. This is an open-access article distributed under the terms of the Creative Commons Attribution License (CC $B Y)$. The use, distribution or reproduction in other forums is permitted, provided the original author(s) and the copyright owner(s) are credited and that the original publication in this journal is cited, in accordance with accepted academic practice. No use, distribution or reproduction is permitted which does not comply with these terms. 